\section{Novel nucleosome regulator}

Post-translational modifications of histone can positively or negatively regulate gene activity, but the full scope of such modifications and their functions are still being determined. In eLife, Schneider and colleagues use mass spectrometry to identify a previously uncharacterized form of histone modification: acetylation of histone $\mathrm{H} 3$ at Lys64 (H3K64ac). H3K64ac associates with euchromatin and the transcriptional start sites of active genes and opposes its repressive counterpart, the trimethylated histone $\mathrm{H} 3 \mathrm{~K} 64 \mathrm{me} 3$. Acetylation of H3K64 occurs on the lateral surface of the histone octamer and is facilitated by the coactivator p300-CBP. The addition of H3K64ac results in destabilization of the nucleosome and gene transcription. This activation mark is found in diverse cell types, including lymphoid cells, and may therefore have important and widespread roles in controlling gene transcription.

eLife (25 March 2014) doi:10.7554/eLife.01632

\section{The protein economy of a cell}

It remains unclear if cells control the stoichiometry of the subunits of various protein complexes through tight control of protein synthesis or through differences in the degradation of excess protein. In Cell, Weismann and colleagues use ribosomal profiling to quantitatively measure absolute protein-synthesis rates and estimate absolute protein copy numbers in a cell. The authors show that proportional synthesis ('tuning' synthesis rates to subunit stoichiometry) is used for more than 90\% of protein complexes in Escherichia coli, which uses polycistronic mRNAs, and in Saccharomyces cerevisiae, in which protein subunits are encoded on different mRNAs. In E. coli, proportional synthesis is achieved through translational control rather than transcriptional control. Because of the different molecular mechanisms for prokaryotic and eukaryotic protein expression, proportional synthesis might be a result of convergent evolution. This study also provides insight into the principles of expression for components of functional modules, such as enzymes of metabolic pathways and transcription factors. Cell 157, 624-635 (2014)

\section{IncRNA for DCs}

Long noncoding RNAs (IncRNAs) have been linked to the regulation of chromatin structure, gene expression and mRNA translation; however, IncRNAs might serve other regulatory roles in the cell. In Science, Wang et al. describe a distinct regulatory mechanism for Inc-DC, a IncRNA that is expressed exclusively in conventional dendritic cells (DCs) and is induced when monocytes differentiate into DCs. Inc-DC is required for the functional presentation of antigens and T cell-priming activities, as well as for the expression of IL-12 by DCs. RNA-mediated interference of Inc-DC results in diminished expression of hundreds of DCassociated genes, which suggests its regulatory role might differ from that of previously described IncRNAs. Instead, Inc-DC is associated with the transcription factor STAT3 in the cytoplasm. Loss of Inc-DC leads to less phosphorylation of STAT3 at Tyr705, whereas ectopic expression of Inc-DC leads to more of this phosphorylation. Inc-DC seems to maintain activated STAT3 by protecting against dephosphorylation by the phosphatase SHP-1, which indicates a unique function for IncRNAs. Whether Inc-DCmediated regulation might also explain the distinct roles of STAT3 in various contexts deserves further investigation.

$L A D$ Science 344, 310-313 (2014) type I interferon signaling, is induced through the phosphorylation of serine and threonine residues in the transactivation domain of IRF3, followed by dimerization of IRF3 and its translocation to the nucleus. In Immunity, Xie and colleagues show that deactivation of IRF3 after activation induced by the RNA helicase RIG-I, TLR3 or TLR4 is regulated through dephosphorylation by the phosphatase PP2A. The interaction between IRF3 and PP2A is mediated by the adaptor RACK1, which mediates the formation of an IRF3-RACK1PP2A complex and promotes the dephosphorylation of IRF3 at steady state. Sendai virus and ligands of TLR3 and TLR4 induce transient dissociation of IRF3 from this complex and translocation of IRF3 to the nucleus. Although high viral titers trigger degradation of IRF3, stimulation with a low titer of virus and TLR ligands induces dephosphorylation of IRF3, which suggests that cells recycle this transcription factor. Immunity 40, 515-529 (2014)

\section{Daxx the downregulator}

The multifunctional nuclear protein Daxx is involved in apoptosis and development, but a role for Daxx in innate immunity has not been clearly established. In the Journal of Biological Chemistry, Cao et al. find that Daxx has broad and high expression throughout the immune system. Macrophages stimulated with certain agonists of Toll-like receptors (TLRs) upregulate their expression of Daxx mRNA and protein. Knockdown of Daxx in macrophages boosts the production of interleukin 6 (IL-6) but seems to leave other proinflammatory cytokines, such as IL- 12 or interferon- $\beta$, intact. After macrophages are stimulated, Daxx is mobilized selectively to the proximal promoter of Il6, where it recruits the histone deacetylase HDAC1. Daxx therefore seems to be a selective negative regulator of inflammation that acts through epigenetic modification of the Il6 locus.

J. Biol. Chem. 289, 9372-9379 (2014) 